\title{
A Review on Barriers to Sustainable Telemedicine Implementation in Ethiopia
}

\section{Getu Gamo Gamo Sagaro ( $\nabla$ getugamo.sagaro@unicam.it )}

Universita degli Studi di Camerino https://orcid.org/0000-0002-5983-0266

GOPI BATTINENI

Universita degli Studi di Camerino

FRANCESCO AMENTA

Universita degli Studi di Camerino Scuola di Scienze del Farmaco e dei Prodotti della Salute

Research article

Keywords: telemedicine, telehealth, m-health, e-health, barriers

Posted Date: October 1st, 2019

DOI: https://doi.org/10.21203/rs.2.15424/v1

License: (1) This work is licensed under a Creative Commons Attribution 4.0 International License.

Read Full License 


\section{Abstract}

Background Different studies showed that the use of telemedicine is effective in reducing hospital burden, suffer from patients, need of transports, hospital fear, save the money and time, and increasing the quality of healthcare. However, the implementation of telemedicine countenances different challenges in developing countries generally and in Ethiopia, particular.Objective This review aims to evaluate barriers which affecting sustainable telemedicine implementation in Ethiopia through analyses of the published works since 2010.

Methods We conducted this review by extracting data from different databases, including MEDLINE, PubMed Central, CINAHL, Google Scholar, Academic Search Ultimate, and Africa-Wide Information. We analyzed finally 12 articles from the above databases based on our eligibility criteria and identified different barriers found in studies. We followed the Preferred Reporting Items for systematic review and meta-analyses (PRSIMA 2009) checklist for this review.

Results We identified 23 barriers through 11 articles and classified barriers into organizational, users, and staff and programmers' barriers. Accordingly, organizational, users', and staff and programmer barriers were $11(48 \%), 7(30 \%)$ and $5(22 \%)$, respectively with the frequency of occurrence through 11 articles and the results organized into table and figure

Conclusions Infrastructure and cost were the most frequently reported barriers, and staff resistance to change was also the critical factor. Inadequate availability Infrastructure and resistance to change could be overcome through training, creating awareness, strengthening Medicare reimbursement regulations and health care insurance programs, allocating budget for new technology and purchase, strong support and follow up, change management techniques.

\section{Introduction}

Telemedicine is defined as the use of electronic communication and information technologies to provide healthcare services when actors are in different locations. We use the World Health Organization (WHO) definition of telemedicine: "The delivery of health care services, where distance is a critical factor, by all health care professionals using information communication technologies (ICT) for exchange valid information for diagnosis, treatment, and prevention of diseases and injuries, research and evaluation and for continuing education of health care providers, all in the interest of advancing the health of individuals and their communities"[1]. As per the WHO, which are not distinguishing between telemedicine and telehealth, and we use the word telemedicine to refer to telemedicine or telehealth for this systematic review[1].

Telemedicine can provide a significant contribution to the health industry, in developed countries, and allows: 1). Real-time communication which connects patients with health care providers through video conferences, home-health monitoring devices, and telephone 
at any time and anywhere, 2). Store and forward, which refers to share images, data, voice, or video and other medical information with physicians for diagnosis across the longdistance, 3). Remote patient monitoring to monitor patient's health parameters and to collect different health-related data while clients are at home and sending their health information's for monitoring, evaluation, and response[1], [2]. Moreover, telemedicine plays a critical role in solving the healthcare access problem in remotely located patients. This is true primarily in the maritime environment where telemedicine allows to provide healthcare and advice for sailing seafarers[3], [4]. Telemedicine also expected in developing countries to reduce the burden of hospitals, suffers from patients, need of transports, hospital fear, save the money and time, and increase the quality of healthcare[5]. Despite, the implementation of telemedicine still difficulties in developing countries where studies reported that nearly $90 \%$ of telemedicine projects were relinquished or failed in developing countries[5][6]. This study will review the present status of telemedicine in Ethiopia and will discuss barriers to sustainable implementation of this technology in the country.

Ethiopia is one of the East Africa countries and located in the horn of Africa, bordered by Kenya, South Sudan, Sudan, Somalia, Djibouti, and Eritrea. Ethiopia has an estimated total population 110,175,175 (as of Thursday, July 11, 2019, based on the latest United Nations estimates), which ranks $12^{\text {th }}$ in the world and the most populous country in Africa next to Nigeria. The population density in Ethiopia is 285 people per square miles; the total land area is 386,102 sq. Miles[7]. Besides, $79 \%$ of the total population live in rural $(87,038,388$ people in 2019)[7]. The median age is 18.8 years, and life expectancy at birth women 65 and men 62. The neonatal, infant, and under-five mortality rates are 29, 48, and 67 per 1,000 live births, respectively, and maternal mortality rate 412 per 100, 000 women in 2016[8].

Sixteen years ago, Ethiopia started expanding access to primary health care through community health extension program and health center[9]. Despite, there is a problem of healthcare service access, especially in the remote or rural area, because of high population growth rate, increased the demand for health care, slow economic growth, and rising health costs. An additional problem is the shortage of medical specialist. This requires that patients travel long-distance where specialists are located. On the other hand, the waiting time for treatment after arriving may take more than a week. Inadequate transportation and hard to reach geographical locations make quite difficult access to 
healthcare services in Ethiopia, where more than $79 \%$ of the population live in rural areas. An option to allow access to healthcare services in a developing country such as Ethiopia is implementing telemedicine services and ensuring sustainability.

Ethiopia adopted a national e-Health strategy in 2014 and identified five main areas for eHealth implementation[10]: 1). Health information system, 2). Telemedicine, 3). M-Health, 4). E-Learning (health workforce training) 5). Community information. However, elearning, especially health information technicians training and allocating at health centers and hospitals are promising but still too young. Some of the other strategies are occasionally implementing, and others are not implemented yet in practice. This review aimed to evaluate the factors affecting the sustainable implementation of telemedicine in Ethiopia through analyses of the published studies since 2010. The result of this review could help authorities to understand different bottlenecks in telemedicine implementation, to prioritize the problems, and to take appropriate measures.

\section{Methods}

A systematic review was undertaken following the checklist from Preferred Reporting Items for systematic review and meta-analyses (PRSIMA 2009)

\section{- Searching Strategy}

To identify the relevant studies, different electronic databases were searched, including MEDLINE, PubMed Central, CINAHL, Google Scholar, Academic Search Ultimate, and Africa-Wide Information. Searches of the internet were carried out using different search engines, and performed between 24 to 28 June 2019. The keywords used for searching in this review were "Barriers" AND "Implementation" OR "Adoption" AND "Telemedicine" OR "e-Health" OR "mHealth" OR "telehealth" OR "mobile health" AND “Ethiopia." We identified 34 potentially relevant articles from the above databases and articles were filtered using the publications dates ranging from the year of 2010 to 2019 to evaluate the barriers to implementing telemedicine and telehealth services in Ethiopia.

\section{- Inclusion and exclusion criteria}

The inclusion criteria of the studies were studies published in the English language, Publication dates and free full-text, and descriptive and cross-sectional studies reporting barriers to implementing telemedicine and telehealth in Ethiopia. We also included Interventional research and narrative analysis, which gave information on the implementation of telemedicine and telehealth in Ethiopia. The assessment of the title and abstracts was performed by three (GG. S, GB, and FA) reviewers independently. The full 
paper was retrieved after the approval of reviewers based on the selection criteria and the entire article was again assessed separately. Finally, data were extracted by two reviewers into structured summary tables and checked by a third reviewer.

Based on our inclusion criteria, overall 11 articles were selected for systematic literature review and three (GG. S, GB, and FA) reviewers read each article and made notes to identify barriers.

\section{- Quality Assessment}

To evaluate the quality of selected studies, the critical appraisal tool for descriptive and cross-sectional studies was used. The critical appraisal tool has 11 questions which adapted from Guyatt GH et al[11], and the questions broadly categorized into three parts, including are the results of the study valid? What are the results? And will the results help locally?

\section{- Data extraction}

The following data were extracted from selected studies: 1). Name of the first author, 2). Publication year, 3). Study design, and 4). Types of barriers. Microsoft (MS) Excel spreadsheets was then used to enter extracted data. Accordingly, we identified different barriers which affect the implementation of telemedicine and telehealth in Ethiopia and organized extracted data into a table.

\section{Results}

Overall 34 potentially relevant studies were selected and of which 30 studies remained after removal of duplicates. Ten studies were rejected by screening abstract and title. Based on our inclusion criteria, three reviewers reviewed the full-length of articles, and in this stage, nine studies were excluded. Finally, we were reviewed 11 articles related to the significant barriers to implementing telemedicine and telehealth services in Ethiopia (Figure 1). Reviewers identified 23 different barriers, and the results are described in table1[2], [9], [19], [10], [12]-[18]. The identified barriers were categorized into user's barriers, organization barriers, and staffs and programmers barriers. Accordingly, unawareness, socioeconomic status, culture, bandwidth, level of education, no phone and eHealth literacy are user's barriers, organization barriers are cost, reimbursement, ICT infrastructure, ehealth equipment, Implementation model, e-health policy, Electricity, skilled human resources, poor internet connectivity, lack of technical assistance and poor supportive system, and staffs and programmers barriers including technically challenged staff, language, poor design, anxiety and resistance to change. According to the reviewer's classification, organizational barriers in number 11 and accounts $48 \%$ of the total frequency of the occurrence of barriers identified and customer, and staffs and programmers' barriers represents 30\% and 22\%, respectively (Figure 2). 
Six studies identified inadequate ICT infrastructure, including poor internet connection, ehealth equipment and insufficient access of electric power for proper implementation of telemedicine and telehealth services, which were considered as the most common organizational barriers[2], [10], [14], [17]-[19]. 
Figure 1: PRISMA flow chart of literature showing the search process, the reasons for exclusion of studies, and the final number of articles included in the review.

Figure 2. Frequency of barriers

The second category was users' barriers. Six studies mentioned main consumers barriers affecting the adoption of telemedicine or telehealth services which are limit of computer or e-Health literacy, the bandwidth of dwelling, access of mobile phone, socioeconomic factors, cultural factors and unawareness of the existence of telehealth products and services[2], [9], [12], [14], [16], [17]. The educational level of the consumer has a high impact on the acceptance of telemedicine services as well as the socioeconomic status influences the access to a cell phone in Ethiopia. Two studies reported that culture affecting the adoption of telehealth services[14], [16], because still there is a challenge in rural communities related to women's healthcare decision, they don't decide alone about their healthcare and to use e-Health services even to participate in awareness programs including meeting and discussion without permission. This is true for married women. Cultural factor is the main challenge to adopt the telehealth services in a remote area, and it will cause the lack of confidence in consumers, and then it to be challenging to convince them to receive health care services without visiting a health care professional. 
The third category was staffs and programmers' barriers, which accounts $22 \%$ of frequency among described barriers. These include technically-challenged staffs, resistance to change, language problem, poor design, and anxiety[2], [9], [14], [15]. Three studies reported that the healthcare professional's resistance to the adoption of telemedicine and telehealth services in Ethiopia was the main influencing factor to successful service implementation[2], [9], [20]. If physicians consider that telemedicine would reduce autonomy and undermine their privileges, the result of this technology would be affected. After appraising losses of some incentives and considering their professional autonomy, health professionals perceived telemedicine as a threat. According to some studies report, healthcare professional tends to become nervous when they are requested to use telemedicine, and their anxiety results in a negative attitude about telemedicine services, and they are likely to engage in resistance[20]. Healthcare professional resistance to change maybe because of the lack of reliable reimbursement system for healthcare delivery through telemedicine in Ethiopia because the providers who invested telemedicine were engaged about reimbursement and taking a return on their investment[21]. On the other hand, telemedicine service implementation requires significant changes to the existing workflow, so many staffs, and providers must invest their time in training new workflows and techniques. This may influence efficiency and effectiveness of the successful telemedicine service establishment.

Medhanyie et al. (2015) and Xue Y et al. (2015) reported that technically-challenged staffs are critical barrier next to resistance to change to the successful implementation of telemedicine and to ensure the sustainability of services in developing countries including Ethiopia[9], [20]. Moreover, two studies reported that limitation of language was another barrier affecting users friendly operate the system. This implies that the consumer loses their confidence in adopting telehealth services at the local level.

Table 1. The selected articles are mentioned in the table with the first name of the author, publication year, study design, and types of barrier. 


\begin{tabular}{|c|c|c|c|}
\hline $\begin{array}{l}\text { Name of First } \\
\text { Author }\end{array}$ & $\begin{array}{l}\text { Publication } \\
\text { Year }\end{array}$ & Study Design & Barriers \\
\hline $\begin{array}{l}\text { Kirubel } \\
\text { Biruk[12] }\end{array}$ & 2018 & $\begin{array}{l}\text { Cross- } \\
\text { sectional }\end{array}$ & Unawareness \\
\hline $\begin{array}{l}\text { Catharina } \\
\text { Barkman[13] }\end{array}$ & 2017 & $\begin{array}{l}\text { Descriptive } \\
\text { case report }\end{array}$ & Reimbursement, infrastructures, cost \\
\hline $\begin{array}{l}\text { Fassil } \\
\text { Shiferaw }[14]\end{array}$ & 2012 & $\begin{array}{l}\text { Descriptive } \\
\text { case report }\end{array}$ & $\begin{array}{l}\text { Cost, e-health policy, implementation model, } \\
\text { ICT infrastructure, language, unawareness, } \\
\text { socioeconomic status, culture, poor telecom } \\
\text { connection, bandwidth of dwelling }\end{array}$ \\
\hline $\begin{array}{l}\text { Araya Abrha } \\
\text { Medhanyie[9] }\end{array}$ & 2015 & $\begin{array}{l}\text { Follow-up } \\
\text { observational } \\
\text { study }\end{array}$ & $\begin{array}{l}\text { Health system, resistance to change, e-health } \\
\text { equipment, unawareness, language, time } \\
\text { consuming, poor application design and } \\
\text { technically-challenged staffs. }\end{array}$ \\
\hline Yajiong Xue[20] & 2015 & $\begin{array}{l}\text { Cross- } \\
\text { sectional }\end{array}$ & $\begin{array}{l}\text { Weak government support, resistance to } \\
\text { change, anxiety, costs }\end{array}$ \\
\hline $\begin{array}{l}\text { Rosalind } \\
\text { Steege[16] }\end{array}$ & 2018 & & $\begin{array}{l}\text { Costs, level of education, culture, no phone, } \\
\text { unawareness }\end{array}$ \\
\hline $\begin{array}{l}\text { The Federal } \\
\text { Ministry of } \\
\text { Health } \\
\text { Ethiopia[10] }\end{array}$ & 2014 & & $\begin{array}{l}\text { Costs, Electricity, Infrastructures and } \\
\text { communication, Human resources (IT, } \\
\text { Informatics, HIT and other professions), } \\
\text { leadership and governance. }\end{array}$ \\
\hline $\begin{array}{l}\text { Kimberly } \\
\text { Harding[17] }\end{array}$ & 2019 & $\begin{array}{l}\text { Descriptive } \\
\text { case report }\end{array}$ & $\begin{array}{l}\text { Electricity, eHealth literacy, Health system, } \\
\text { telecommunication service, cost, Internet } \\
\text { connection, no phone, trained human } \\
\text { resources }\end{array}$ \\
\hline $\begin{array}{l}\text { BELAY TAMIRU } \\
\text { SHITA[18] }\end{array}$ & 2013 & $\begin{array}{l}\text { Cross- } \\
\text { sectional }\end{array}$ & $\begin{array}{l}\text { Cost, culture, Internet connection, system } \\
\text { unreliability, unawareness, lack of technical } \\
\text { assistance, }\end{array}$ \\
\hline $\begin{array}{l}\text { Amare Ayalew } \\
\text { Abera[19] }\end{array}$ & 2014 & $\begin{array}{l}\text { Cross- } \\
\text { sectional }\end{array}$ & $\begin{array}{l}\text { Cost, availability of technical support, Internet } \\
\text { connection, ICT infrastructure availability, } \\
\text { availability of technological infrastructure. }\end{array}$ \\
\hline $\begin{array}{l}\text { TsegazaabT. } \\
\text { Weldegebrial[2] }\end{array}$ & 2017 & Case study & $\begin{array}{l}\text { Cost, Electricity, availability of Infrastructure, } \\
\text { Internet connection, unawareness, leadership, } \\
\text { resistance to change }\end{array}$ \\
\hline
\end{tabular}




\section{Discussion}

\section{Summary of main findings}

According to this review, the most frequently reported barriers to the implementation of telemedicine services are cost and infrastructure[2], [9], [10], [13], [14], [16]-[20]. The initial cost of establishing telemedicine service is very high and to function telemedicine services in rural or remote area for underserved communities needs telecommunication expenses, training (health personnel and patients), needs for new advanced technologies and electric power supplies. Fifteen years ago, in Ethiopia, all concerned bodies like universities, telecommunication authority, the ministry of health, policymakers, and other responsible institution agreed to support telemedicine implementation[22]. However, the each of some services such as inadequate access to electricity power (only $8 \%$ of rural household have access in 2016)[8], lack of e-Health literacy, limited access to health IT to address gaps in health IT literacy for Health Extension Workers (HEWs)[17], Poor telecommunication and internet connectivity[14], [17]-[19], and shortage of health information technicians (HITs) were the major bottlenecks to the implementation of telemedicine services in Ethiopia. We recommend that Ethiopian government should focus on organizational barriers especially infrastructure challenges such as unstable power supplies, poor communication network, inadequate internet connectivity with limited bandwidth, lack of human resources with necessary technical expertise to successfully implement the telemedicine and to ensure its sustainability. This is most of the healthcare institutions, telecommunication, and electricity infrastructures are owned and controlled by the government. Moreover, the government should invite other stakeholders like nongovernmental organizations to support technical issues as well as financial resources at the initial phase.

On the other hand, culture[14], [16], [18] and staff resistance to change[2], [9], [20] are the critical factor affecting telemedicine implementation in developing multicultural countries like Ethiopia. Adoption of telemedicine services requires the acceptance of users involved in the process. This review may indicate that the unawareness of, comfort in, the use of telemedicine services because the willingness of change and adhere to new services is dependent on the culture of users, organizations and providers (employees). Many healthcare providers especially physicians consider that the use of telemedicine will make physician-patient communication ineffective and implementation of telemedicine may alter their current work practices[20], [23], [24]. This negative perception leads health professionals to resistance to telemedicine adoption. As a result, national and local level 
advocacy on the benefit and the proper use of telemedicine services, as well as individual training, would help to address fears and resistance and increase acceptance among health professionals and users or patients correspondingly. The Medicare reimbursement regulations should be strengthening, and applied properly to encourage for the health professional to participate in telemedicine modalities of care. From the side of users, sometimes rural people hard to believe that they can get healthcare services without having face-to-face communications. Some efforts are necessary to make them aware of the importance of this technologies. Governments should take the responsibility to change cultural behaviors as well as unawareness by public education.

The language is also another barrier[9], [14] because users with no technical supporter, the limited language knowledge is one of the factors to use-friendly the system. Ethiopia is multilingual country, considering local languages during the design phase is essential for overcoming this barrier to successful implementation and enabling diffusion of telemedicine services. Technical solution should be considered related to language issues at the programmer level. Sometimes users and providers encounter the technology itself can be a challenge[9], [17] because some users or providers may not be as familiar with the functionality of their mobile devices, internet connection or telemedicine applications. It would be crucial that technical supporters offer guidance for the successful implement and to ensure the confidence of users at the local level.

\section{Conclusions}

Infrastructure and cost are the most frequently reported barriers in this review. In other words, $91 \%$ of the selected articles reported cost and infrastructures were the critical barriers to telemedicine implementation in Ethiopia. Besides, staff resistance to change also reported as a factor affecting the successful implementation of telemedicine. Inadequate availability infrastructure especially ICT infrastructures such as computer, electricity power supply, Internet connection, and inadequate health information technicians (HIT) could be overcome through training, repair and replacing, allocating budget and purchase new technology, enhancing access of power supply, and internet network and connection. Regarding expenditure, including the initial cost for the establishment, telecommunication expenses, training service providers, and patients' expenses could be covered by the government and by other stakeholders like non-governmental organizations through the government. In this respect, the Federal Ministry of Health and telecommunication authority should work closely with each other to integrate health information with the development of ICT.

Ethiopia Federal Minister of Health (FMoH) should give attention on policy and e-health strategies to outline the visions and objectives regarding applications, provisions, control, and standards related to telemedicine solutions and focuses on the integration of e-health 
strategies with national health policy. The incorporation of e-health strategies with national health policy may help to facilitate and enhance the chance of successful and sustainable implementation telemedicine services by providing a structural frame and protocol for planning and development of services. In general, this study provides directions to the government to prioritize the problems and to develop appropriate solutions.

\section{Declarations}

\section{Declaration of Conflicting of Interests}

The authors declared that no potential conflicts of interest with publications of this article.

\section{Funding}

The authors received no finical support for the research, authorship, and/or publications of this article.

\section{Author's Contributions}

Getu Gamo Sagaro: has conceived the study, participated in the design of the study, collected published studies from different databases by searching, performed literature review, data extraction, and descriptive statistical analysis, and drafting the manuscript for relevant intellectual content. Gopi Battineni: has involved in the design of the study and collection of published work, participated in data extraction, performed literature review, participated in paper writing. Professor Francesco Amenta: has conceived and planned the study, guided overall stages of the study, revisited the manuscript critically for important intellectual content, involved in reviewing and editing the manuscript. We confirm that the manuscript has been read and approved by all named authors before submission.

\section{References}

[1] World Health Organization, "A health telematics policy in support of WHO's Healthfor-all strategy for global health development: report of the WHO Group Consultation on Health Telematics, 11-16 December, Geneva," 1997.

[2] T. Weldegebrial, "Telehealth in Ethiopia - The Barriers Vs . The Success Factors," no. December 2016, 2017.

[3] E. Çakır and Ö. Arslan, "Turkish Telemedical Assistance Service: last four years of activity," Int. Marit. Health, vol. 69, no. 3, pp. 184-191, 2018. 
S. S. Mahdi and F. Amenta, "Eighty years of CIRM. A journey of commitment and dedication in providing maritime medical assistance," Int. Marit. Health, vol. 67, no. 4, pp. 187-195, 2016.

[5] Surya Bali, "Barriers to Development of Telemedicine in Developing Countries," Intech, vol. i, no. tourism, p. 13, 2016.

[6] A. Alaboudi, A. Atkins, B. Sharp, A. Balkhair, M. Alzahrani, and T. Sunbul, "Barriers and challenges in adopting Saudi telemedicine network: The perceptions of decision makers of healthcare facilities in Saudi Arabia," J. Infect. Public Health, vol. 9, no. 6, pp. 725-733, 2016.

[7] U. Nations, "Ethiopia Population (2019) - Worldometers." [Online]. Available: https://www.worldometers.info/world-population/ethiopia-population/. [Accessed: 11Jul-2019].

[8] EDHS, FEDERAL DEMOCRATIC REPUBLIC OF ETHIOPIA Demographic and Health Survey. 2016.

[9] A. A. Medhanyie et al., "Health workers' experiences, barriers, preferences and motivating factors in using mHealth forms in Ethiopia," 2015.

[10] The Federal Ministry of Health Ethiopia, "Ethiopian National eHealth Strategic," pp. 1-4, 2015.

[11] Gordon Guyatt and Maureen O. Meade, "User's guides to the medical literature a mannual for evidence-based clinical practice second edition."

[12] K. Biruk and E. Abetu, "Knowledge and Attitude of Health Professionals toward Telemedicine in Resource-Limited Settings: A Cross-Sectional Study in North West Ethiopia," J. Healthc. Eng., vol. 2018, pp. 1-7, 2018.

[13] C. Barkman and L. Weinehall, "Policymakers and mHealth: Roles and expectations, with observations from Ethiopia, Ghana and Sweden," Glob. Health Action, vol. 10, no. 3, 2017.

[14] F. Shiferaw and M. Zolfo, "The role of information communication technology (ICT) towards universal health coverage: The first steps of a telemedicine project in Ethiopia," Glob. Health Action, vol. 5, no. 1, p. 15, 2012.

[15] Y. Xue, H. Liang, V. Mbarika, R. Hauser, P. Schwager, and M. Kassa Getahun, "Investigating the resistance to telemedicine in Ethiopia," Int. J. Med. Inform., vol. 84, no. 8, pp. 537-547, Aug. 2015. 
[16] R. Steege, L. Waldman, D. G. Datiko, A. Z. Kea, M. Taegtmeyer, and S. Theobald, "The phone is my boss and my helper' - A gender analysis of an mHealth intervention with Health Extension Workers in Southern Ethiopia," J. Public Heal. (United Kingdom), vol. 40, pp. II16-II31, 2018.

[17] Kimberly and T. B. M. Harding, Gashaw Andargie Biks, Mulat Adefris, Jordann Kiros Terefe Gashaye, Binyam Tilahun4, Michael Volynski, Shashank Zeleke Abebaw , Kassahun Dessie, "A mobile health model supporting Ethiopia's eHealth strategy," Digit. Med., pp. 54-65, 2018.

[18] B. T. Shita, "Utilization of Telemedicine in Tikur Anbessa," Thesis, no. June, pp. 3-49, 2013.

[19] H. J. Watson, "Assessment of Ethiopian Health Facilities Readiness for Implementation of Telemedicine," Commun. Assoc. Inf. Syst., vol. 34, no. 1, pp. 12471268, 2014.

[20] Y. Xue, H. Liang, V. Mbarika, R. Hauser, P. Schwager, and K. Getahun, "Investigating the resistance to telemedicine in Ethiopia," Int. J. Med. Inform., vol. 84, pp. 537-547, 2015.

[21] C. Scott Kruse, P. Karem, K. Shifflett, L. Vegi, K. Ravi, and M. Brooks, "Evaluating barriers to adopting telemedicine worldwide: A systematic review," J. Telemed. Telecare, vol. 24, no. 1, pp. 4-12, 2018.

[22] M. Kifle, a Solomon, C. Okoli, and V. Mbarika, "Critical success factors for telemedicine in Ethiopia," Innov. Through Inf. Technol. Vols 1 2, vol. 46, no. 8, pp. 426-429, 2004.

[23] S. Bagchi, "Telemedicine in Rural India," PLoS Med., vol. 3, no. 3, p. e82, 2006.

[24] C. Shah and G. Marchionini, "Telemedicine in Sub-Saharan Africa: The Case of Teleophthalmology and Eye Care in Ethiopia," Int. Rev. Res. Open Distance Learn., vol. 14, no. 4, pp. 90-103, 2013.

\section{Figures}




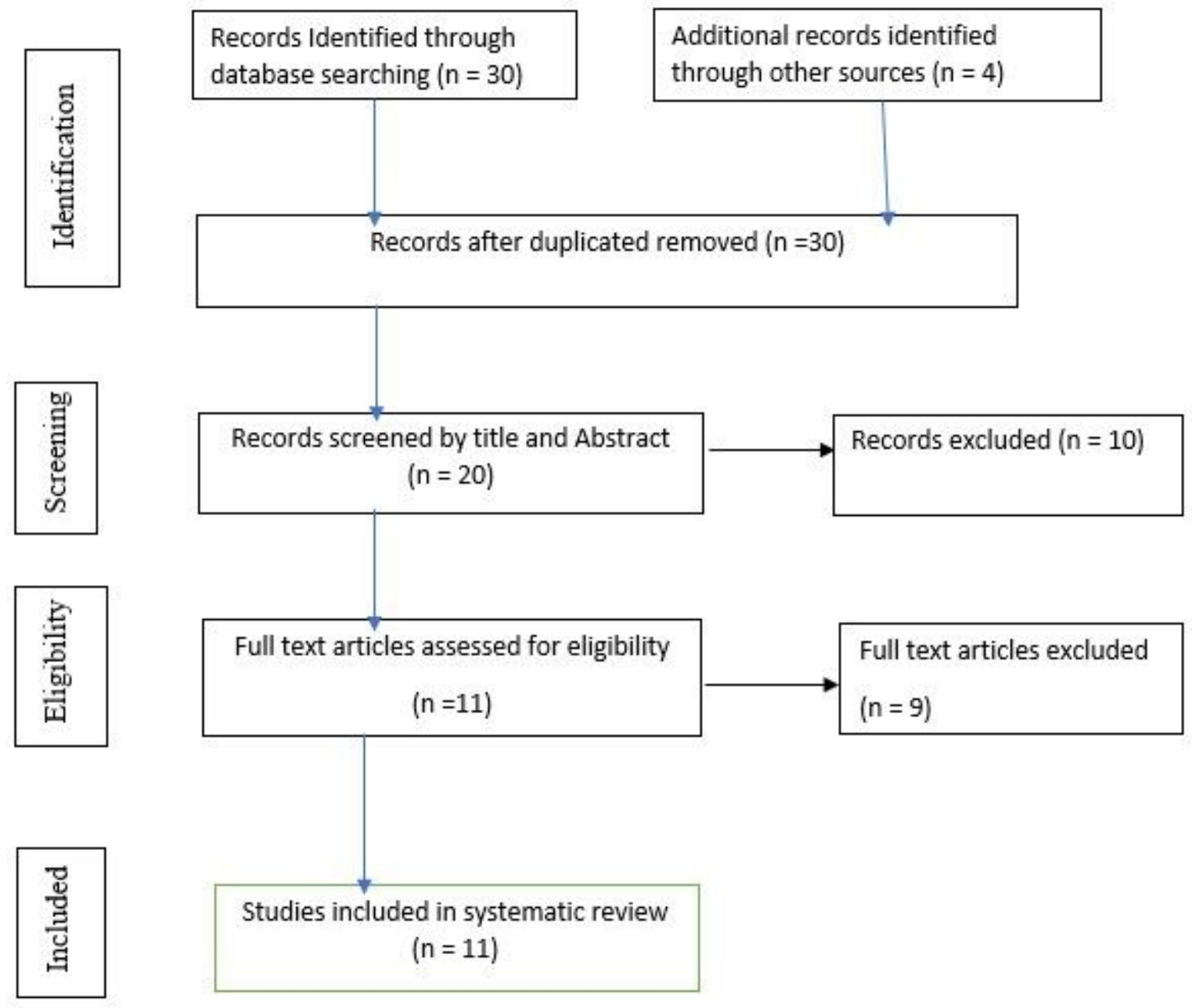

Figure 1: PRISMA flow chart of literature showing the search process, the reasons for exclusion of studies, and the final number of articles included in the review.

\section{Figure 1}

PRISMA flow chart of literature showing the search process, the reasons for exclusion of studies, and the final number of articles included in the review 


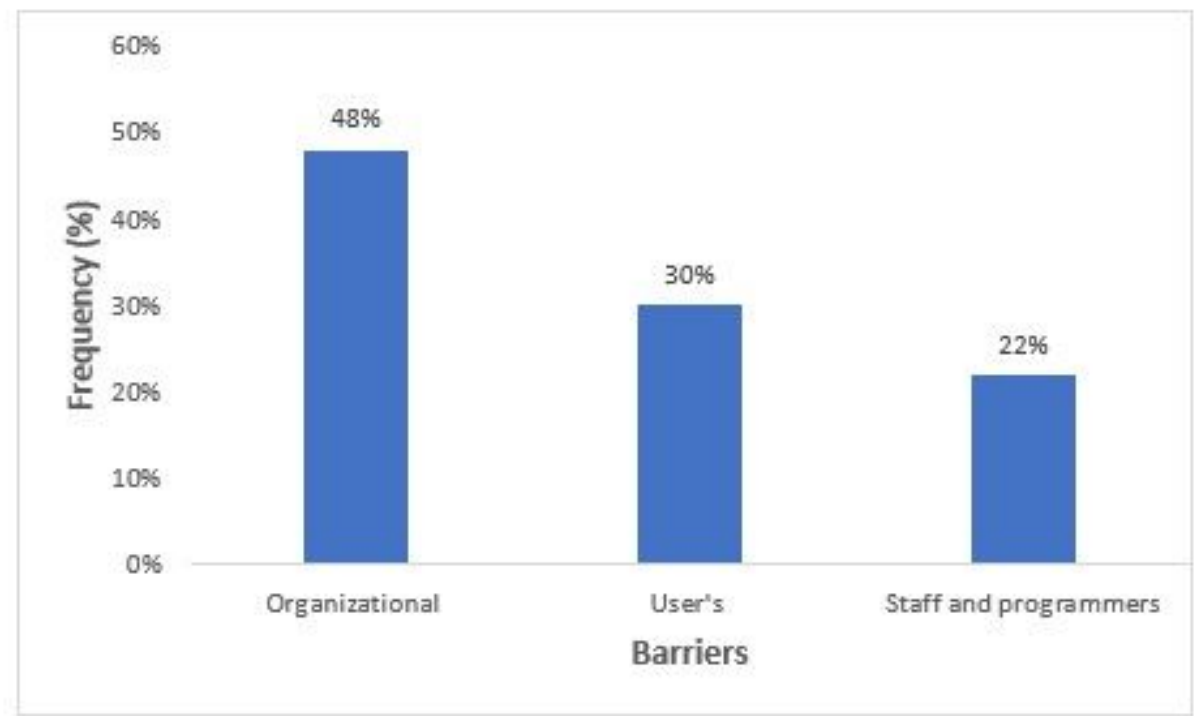

Figure 2. Frequency of barriers

Figure 2

Frequency of barriers 